\title{
SMAC mimetics induce autophagy-dependent apoptosis of HIV-1-infected macrophages
}

\author{
Grant R. Campbell (10), Rachel K. To ${ }^{1,3}$, Gang Zhang ${ }^{1,4}$ and Stephen A. Spector $\mathbb{B}^{1,2}$
}

\begin{abstract}
Human immunodeficiency type 1 (HIV)-infected macrophages (HIV-M $\varphi$ ) are a reservoir for latent HIV infection and a

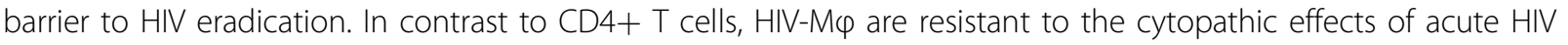
infection and have increased expression of cell survival factors, including X-linked inhibitor of apoptosis (XIAP), baculoviral IAP repeat containing (BIRC) 2/CIAP1, beclin-1, BCL2, BCL-xl, triggering receptor expressed on myeloid cells 1, mitofusin (MFN) 1, and MFN2. DIABLO/SMAC mimetics are therapeutic agents that affect cancer cell survival and induce cell death. We found that DIABLO/SMAC mimetics (LCL-161, AT-406 (also known as SM-406 or Debio 1143), and birinapant) selectively kill HIV-M $\varphi$ without increasing bystander cell death. DIABLO/SMAC mimetic treatment of HIV$M \varphi$-induced XIAP and BIRC2 degradation, leading to the induction of autophagy and the formation of a deathinducing signaling complex on phagophore membranes that includes both pro-apoptotic or necroptotic (FADD, receptor-interacting protein kinase (RIPK) 1, RIPK3, caspase 8, and MLKL) and autophagy (ATG5, ATG7, and SQSTM1) proteins. Genetic or pharmacologic inhibition of early stages of autophagy, but not late stages of autophagy, ablated this interaction and inhibited apoptosis. Furthermore, DIABLO/SMAC mimetic-mediated apoptosis of HIV-M $\varphi$ is dependent upon tumor necrosis factor signaling. Our findings thus demonstrate that DIABLO/SMAC mimetics selectively induce autophagy-dependent apoptosis in HIV-M $\varphi$.
\end{abstract}

\section{Introduction}

Although latently infected $\mathrm{CD} 4+\mathrm{T}$ cells are the predominant human immunodeficiency virus type 1 (HIV) reservoir in infected persons on suppressive antiretroviral therapy (ART), other cell types including macrophages serve as important sites of viral persistence ${ }^{1-3}$. Long-lived HIV-infected perivascular macrophages contain replicationcompetent proviral HIV DNA, support persistent permissive HIV infection in the absence of CD4 $+\mathrm{T}$ cells ${ }^{4,5}$, and contain lower intracellular concentrations of antiretrovirals than CD4+ T cells resulting in ongoing HIV replication ${ }^{6}$. HIV-infected macrophages also harbor and synaptically transmit replication-competent virus to nearby $\mathrm{CD} 4+$

Correspondence: Grant R. Campbell (gcampbell@ucsd.edu) or

Stephen A. Spector (saspector@ucsd.edu)

'Division of Infectious Diseases, Department of Pediatrics, University of California San Diego, La Jolla, CA, USA

${ }^{2}$ Rady Children's Hospital, San Diego, CA, USA

Full list of author information is available at the end of the article

Edited by G. M. Fimia
$\mathrm{T}$ cells, a process that is resistant to antibody-mediated neutralization ${ }^{7}$. Additionally, HIV signals through multiple pathways to reprogram the transcriptome and proteome of human macrophages rendering them resistant to both the cytopathic effects of HIV infection and to CD8+ T-cellmediated killing ${ }^{8-10}$. HIV decreases the expression of proapoptotic proteins, while increasing the expression of antiapoptosis regulatory proteins, including BCL2 family members, and the inhibitor of apoptosis proteins (IAP) $\mathrm{X}$-linked inhibitor of apoptosis (XIAP), baculoviral IAP repeat containing (BIRC) 2 (formerly known as cIAP1), and BIRC3 (refs. ${ }^{11-21}$ ). The overexpression of IAPs can inhibit cell death through a number of mechanisms, including direct inhibition of caspases and ubiquitination of receptor-interacting protein kinase (RIPK) 1, an important convergence point between pro-death, pro-survival, and proinflammatory signals. They may also play a role in the establishment of HIV latency and cytopathogenesis ${ }^{22}$. Thus,

\section{(c) The Author(s) 2020}

(c) (i) Open Access This article is licensed under a Creative Commons Attribution 4.0 International License, which permits use, sharing, adaptation, distribution and reproduction cc) in any medium or format, as long as you give appropriate credit to the original author(s) and the source, provide a link to the Creative Commons license, and indicate if changes were made. The images or other third party material in this article are included in the article's Creative Commons license, unless indicated otherwise in a credit line to the material. If material is not included in the article's Creative Commons license and your intended use is not permitted by statutory regulation or exceeds the permitted use, you will need to obtain permission directly from the copyright holder. To view a copy of this license, visit http://creativecommons.org/licenses/by/4.0/. 
IAP are relevant targets for therapeutic exploitation in HIV cure strategies.

To circumvent the ability of IAP to turn pro-death into pro-survival signals in cancer cells, small-molecule DIA$\mathrm{BLO} / \mathrm{SMAC}$ peptidomimetics (SM) were developed that mimic the $\mathrm{N}$-terminal 4 amino acid IAP-binding sequence of DIABLO. The interaction of SM with XIAP leads to caspase activation $^{23}$, while the binding of SM to BIRC2 and BIRC3 enhances their E3 ligase activity promoting their autoubiquitination and proteasomal degradation with subsequent cell death ${ }^{19,24,25}$. In this study, we examined the ability of the SM LCL-161 (ref. ${ }^{26}$ ), AT-406 $\left(\right.$ ref. ${ }^{27}$ ), and birinapant ${ }^{28}$ to induce selective cell death in HIV-infected macrophages.

\section{Results}

DIABLO mimetics selectively kill HIV-infected macrophages

We previously showed that HIV latently infected CD4+ $\mathrm{T}$ cells $\left(\mathrm{HIV}-\mathrm{T}_{\mathrm{CM}}\right.$ ) have increased expression of BIRC2 and XIAP ${ }^{19}$. Therefore, we examined the expression of BIRC2 and XIAP in HIV $\mathrm{Ba}_{\mathrm{L}}$-infected macrophages (HIV$\mathrm{M} \phi$; Supplementary Fig. 1); XIAP and BIRC2 expression was significantly upregulated in $\mathrm{HIV}-\mathrm{M} \phi(P<0.05$; Fig. 1$)$. As SM induce the rapid degradation of BIRC2 and XIAP in cancer cells and HIV-T ${ }_{\mathrm{CM}}{ }^{19,24,25,29-31}$, we evaluated the ability of the SM LCL-161, AT-406, and birinapant to induce BIRC2 and XIAP degradation in uninfected macrophages and $\mathrm{HIV}-\mathrm{M} \phi$. All three compounds induce significant BIRC2 and XIAP degradation in uninfected cells and HIV-M $\phi$ (Fig. 1a). However, the concentrations required to induce significant degradation are 10-100x lower in HIV-M $\phi$ than in uninfected cells.

SM-induced rapid degradation of BIRC2 and XIAP is a key early event in SM-induced cell death ${ }^{24,25,29-31}$. We evaluated the toxicity of the three SM against both uninfected macrophages and HIV-M $\phi$, using the presence of formamide-sensitive single-stranded DNA (a specific indicator of apoptosis ${ }^{32}$ ) and the release of lactate dehydrogenase (LDH). In uninfected macrophages, LCL-161, AT-406, and birinapant all showed minimal toxicity at the concentrations tested (Fig. 1b). Conversely, all three SM induced a dose-dependent increase in HIV-M $\phi$ cytotoxicity. We observed similar results in $\mathrm{HIV}_{93 \mathrm{IN} 905^{-} \text {-infected }}$ macrophages (Fig. 1c). The increase in HIV-M $\phi$ cytotoxicity corresponded to the dose-dependent proteolysis of poly(ADP-ribose) polymerase 1 (PARP1; a substrate of caspase (CASP) 3) and CASP8 cleavage (Fig. 2a). We then assessed whether the kinetics of killing uninfected macrophages is slower than that observed in HIV-M $\phi$. We observed no significant increase in LDH release in the uninfected cells post-SM treatment over $168 \mathrm{~h}$, indicating that at no point do they undergo biologically significant cell death in response to SM (Fig. 2b). In contrast, $\mathrm{SM}$ induced a time-dependent increase in HIV-M $\phi$ cytotoxicity. Importantly, we measured no SM-mediated increase in HIV p24 antigen release after LCL-161 or birinapant treatment, indicating that the SM were killing $\mathrm{HIV}-\mathrm{M} \phi$ in the absence of increased virus production (Fig. 2c). Conversely, AT-406 treatment induced a dosedependent increase of HIV p24 antigen release.

SM-mediated cell death conventionally requires the presence of pro-apoptotic tumor necrosis factor (TNF) family ligands ${ }^{33,34}$, and we previously demonstrated that SM selectively induce TNF-independent death of HIV$\mathrm{T}_{\mathrm{CM}}{ }^{19}$. Therefore, to determine the role of TNF in SM-mediated death of HIV-M $\phi$, we blocked TNF signaling with a specific neutralizing antibody and observed that neutralization of TNF significantly ablated SM-mediated HIV-M $\phi$ cell death (Fig. 2d), suggesting that, unlike HIV-T $\mathrm{T}_{\mathrm{CM}}$, the SM-mediated killing of HIV$\mathrm{M} \phi$ is TNF dependent.

Although SM induce the proteasomal degradation of IAP, we examined whether they also stimulate autophagy in HIV-M $\phi$. Autophagic flux is assessed by monitoring the biogenesis of autophagosomes through a ubiquitinlike system that involves autophagy-related (ATG) 7 and the ATG12-ATG5 complex that converts cytosolic microtubule-associated protein 1 light chain 3 beta (MAP1LC3B or LC3B)-I to LC3B-II. The ATG12-ATG5 complex then ligates LC3B-II to the nascent autophagosome membrane. The polyubiquitin-binding protein sequestosome 1 (SQSTM1, p62) and SQSTM1-bound polyubiquitinated proteins are incorporated into completed autophagosomes that then fuse with lysosomes, resulting in the degradation of the engulfed components, as well as LC3B-II and SQSTM1 associated with the inner membrane. Thus, the quantification of SQSTM1 and the conversion of LC3B-I to LC3B-II, and its turnover are indicators of autophagy induction and flux ${ }^{35}$. In uninfected macrophages, SM increases LC3B lipidation at the highest concentrations tested while having no significant effect on SQSTM1 degradation, indicating an absence of induced autophagy (Fig. 3a). In contrast, LCL-161 and AT-406 induced a dose-dependent significant increase in LC3B-II lipidation, BECN1 expression, and SQSTM1 degradation. While we observed a change in LC3B lipidation and SQSTM1 expression using birinapant, at no point did we observe an increase in BECN1. To confirm the induction of autophagy in SM-treated HIV-M $\phi$, and not simply the activation of MAP1LC3B and SQSTM1 transcription, we used bafilomycin $A_{1}$, an inhibitor of autophagosome-lysosome fusion. Blots of cell lysates confirmed autophagic flux in $\operatorname{HIV}-\mathrm{M} \phi$, with increased LC3B-II and SQSTM1 accumulation in bafilomycin $\mathrm{A}_{1}$ treated cells relative to vehicle controls (Fig. 4). Crucially, pretreatment with bafilomycin $\mathrm{A}_{1}$ did not reduce the effect of SM-induced XIAP or BIRC2 degradation in HIV$\mathrm{M} \phi$ (Fig. 4b). 


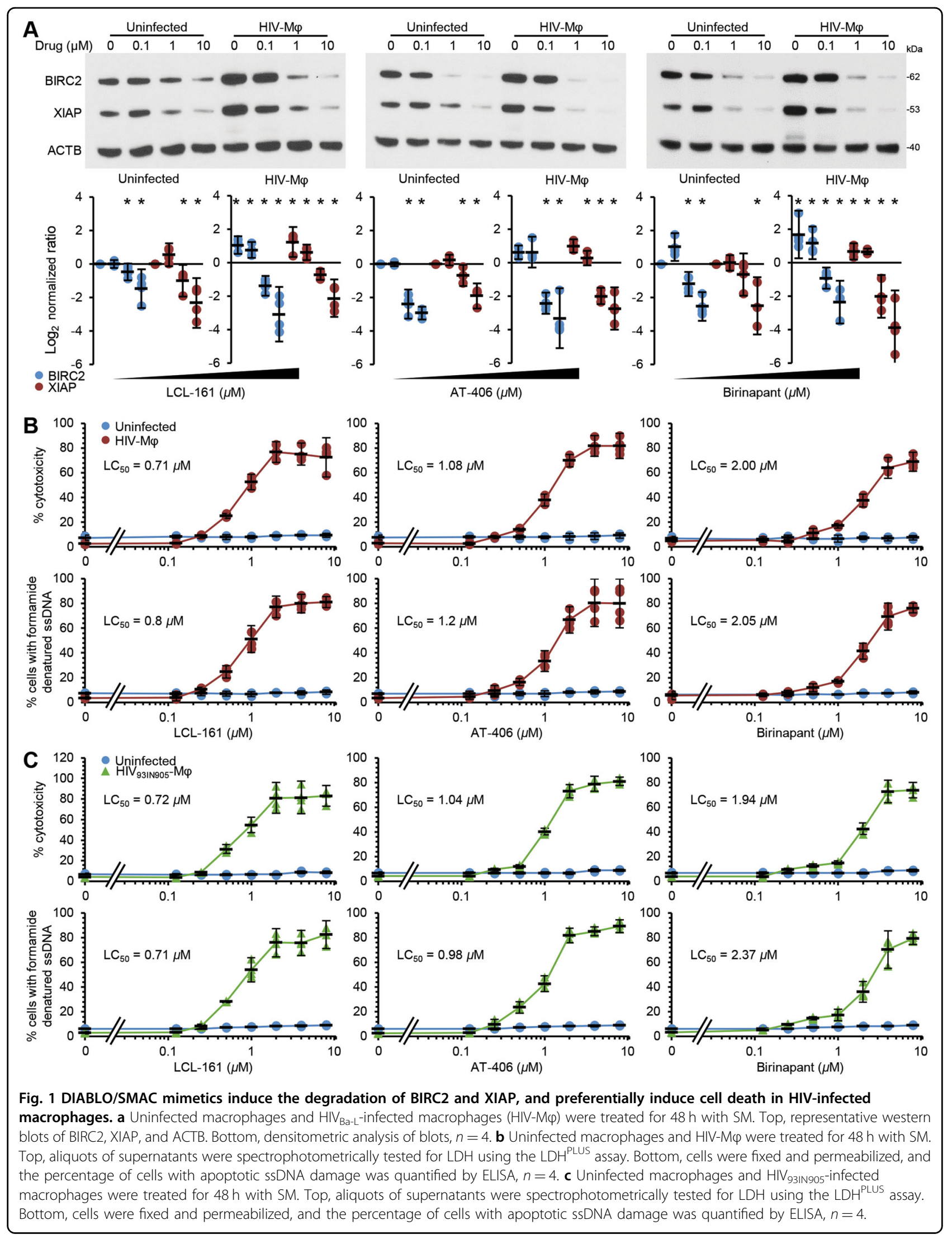




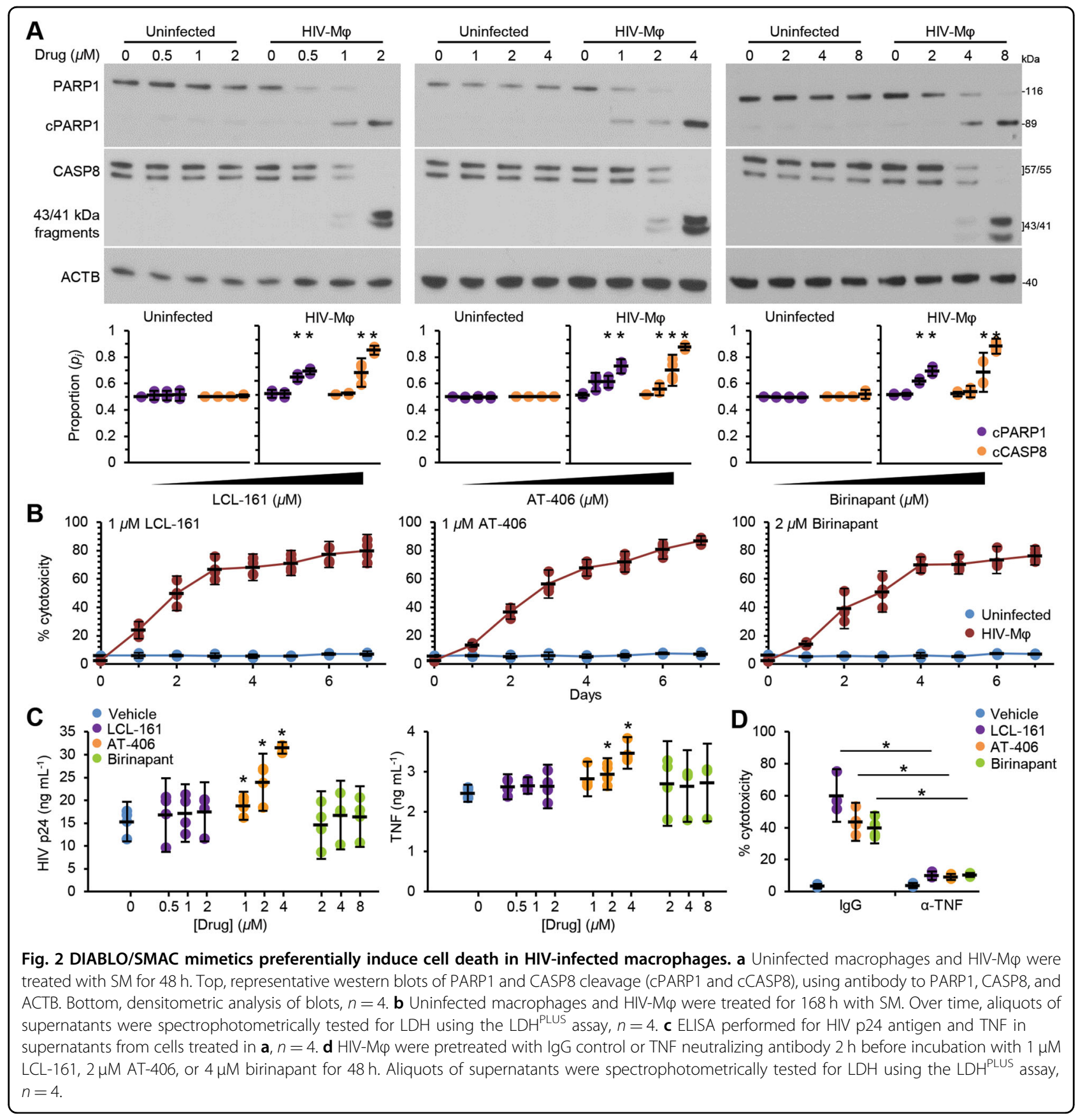

\section{SM induce apoptosis in HIV-infected macrophages}

To determine the mechanism of cell death, we examined the effect of SM on HIV-M $\phi$ in the presence of pharmacological inhibitors of apoptosis (pan-caspase inhibitor z-VAD-FMK), autophagy (PI3K inhibitor wortmannin, bafilomycin $\mathrm{A}_{1}$, and chloroquine, a lysosomotropic agent that prevents endosomal acidification), and/or necroptosis (RIPK1 inhibitor necrostatin-1). None of the HIV-M $\phi$ tested showed a purely apoptotic or necroptotic phenotype in response to SM with neither z-VAD-FMK nor necrostatin-1 alone able to arrest SM-induced cell death
(Fig. 5a). However, co-treatment with both z-VAD-FMK and necrostatin- 1 resulted in an $88.9 \%$ reduction in SMmediated death of HIV-M $\phi$. During necroptosis, the effector mixed-lineage kinase domain-like protein (MLKL) is recruited to the necrosome where it is phosphorylated by RIPK3, inducing a conformational change that causes it to translocate to and permeabilize the plasma membrane $^{36}$. Therefore, we assessed the role for MLKL in SM-mediated cell death. In the absence of z-VAD-FMK, we did not observe significant SM-mediated MLKL phosphorylation despite observing 50-66\% cell death 


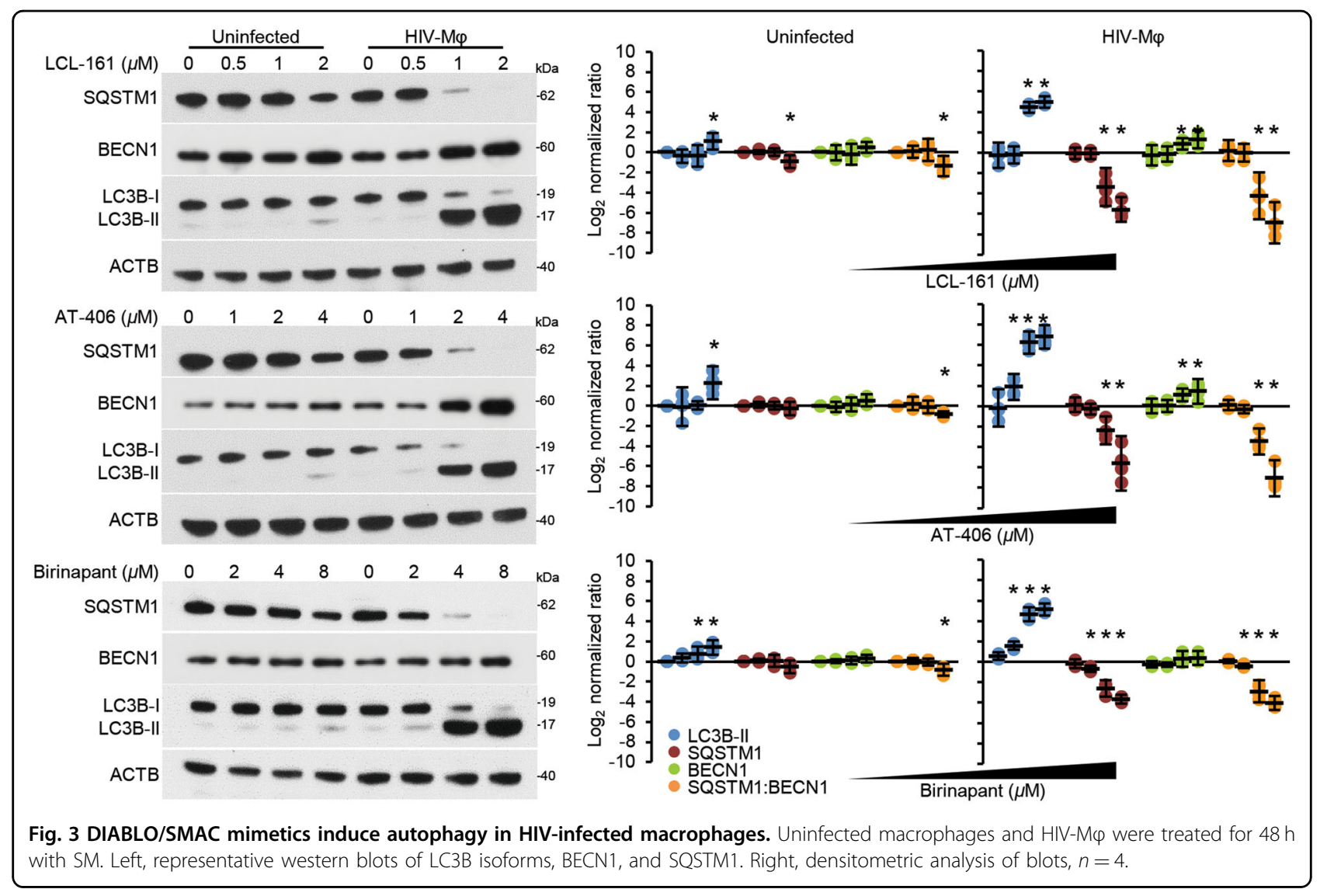

(Fig. 5b). However, when we pretreated cells with z-VADFMK, SM treatment induced significant MLKL phosphorylation, suggesting that cells switched to necroptosis when we inhibited apoptosis.

We then examined how autophagy affects SM-mediated cell death in HIV-M $\phi$. In these experiments, inhibition of the autophagy conjugation cascades with wortmannin or RNA interference (RNAi) for ULK1, ATG5, ATG7, or SQSTM1 (Fig. 5c) led to increased cell viability in the presence of SM (Fig. 5a, d). In contrast, we observed no effect on SM-induced death by blocking autophagic flux using either simultaneous RNAi for $A T G 2 A$ and $A T G 2 B$ $(A T G 2 A / B)$, which causes the accumulation of immature unclosed autophagosomal structures (Fig. 5d), or the inhibition of autophagosome-lysosome fusion using either chloroquine or bafilomycin $\mathrm{A}_{1}$ (Fig. 5a). Importantly, we also observed no effect on SM-induced death with the inhibition of LC3-associated phagocytosis using $R U B C N$ silencing. These data suggest that neither fully formed autophagosomes nor autophagy-mediated degradation of cargo is required to induce cell death in response to SM, as, if this were the case, a similar effect on cell death would occur regardless of autophagy stage inhibited.
We next tested whether varying expression of key components of the apoptotic and necroptotic response might contribute to the differential response to $\mathrm{SM}$ in uninfected macrophages and HIV-M $\phi$. We observed no increase in the expression of RIPK1, RIPK3, FADD, MLKL, ATG5, or ATG7 in HIV-M $\phi$ (Fig. 6a), indicating that HIV-M $\phi$ cell death in response to SM correlates with HIV-induced increased expression of LC3B-II, BECN1, XIAP, and BIRC2. In the next series of experiments, we assessed the effect of SM on these same key players of the apoptotic and necroptotic response. At the doses tested, we observed no significant changes in the cleavage of RIPK1 or RIPK3, or the expression of ATG7, MLKL, ATG12-ATG5, or FADD in uninfected macrophages. Likewise, we did not observe an increase in ATG12-ATG5 or FADD expression in HIV-M $\phi$. Conversely in HIV-M $\phi$, we observed the SM-mediated cleavage of RIPK1 and RIPK3 (Fig. 6a), which is consistent with RIPK1 and RIPK3 being targets of CASP8 cleavage, and thus as CASP8 is activated, RIPK1 and RIPK3 are cleaved $^{37}$. We also observed an increase in the expression of ATG7, MLKL, and FADD in HIV-M $\phi$, suggesting that components of the apoptotic necroptotic and autophagic machinery are involved. 


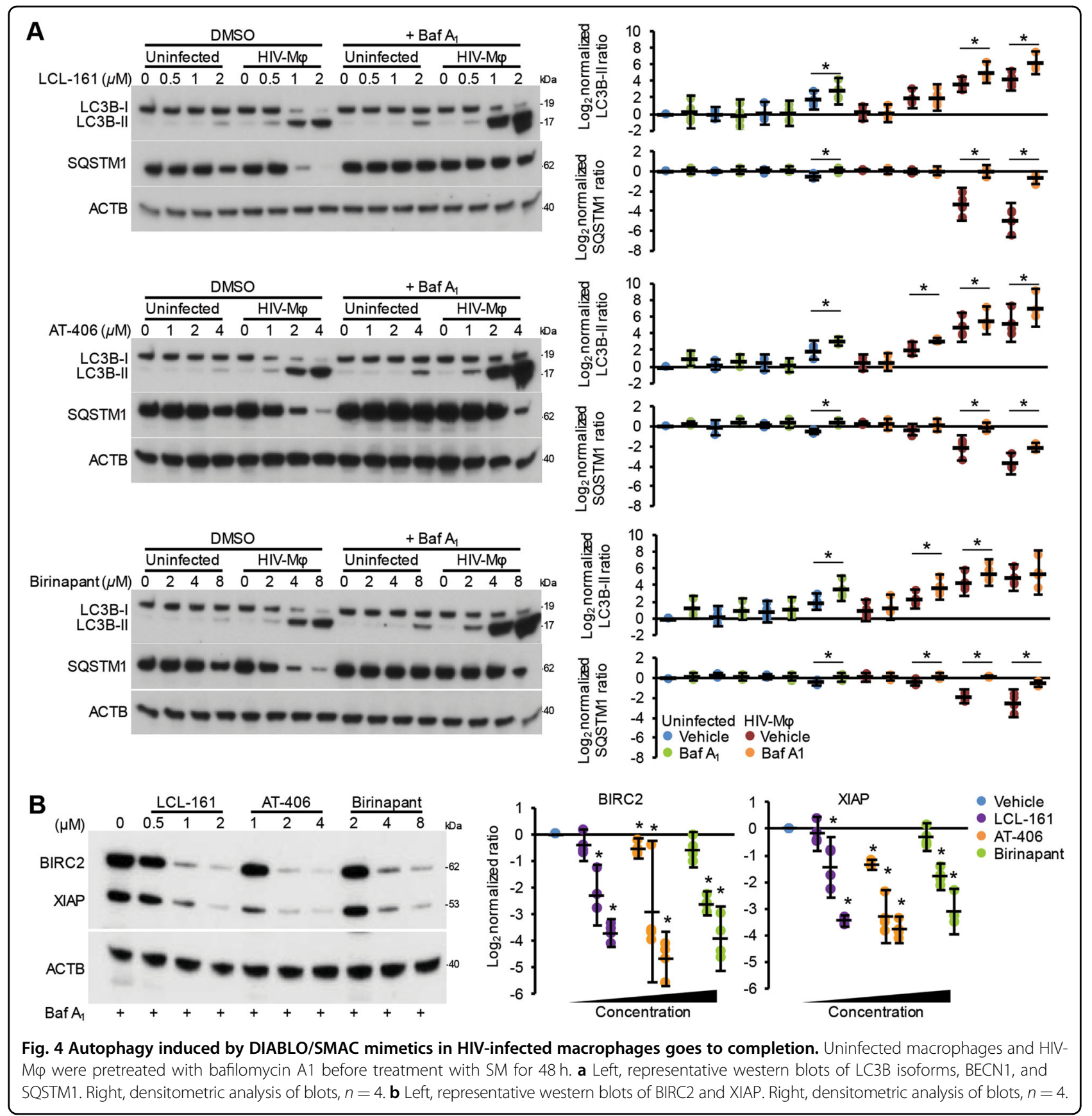

SM-induced apoptosis is dependent upon the autophagy machinery

Following the loss of BIRC2, a death-inducing signaling complex (DISC) involving RIPK1, CASP8, and FADD forms ${ }^{19,38,39}$. Having observed that the induction, but not the completion of autophagy is required for SMmediated killing of $\mathrm{HIV}-\mathrm{M} \phi$, we examined whether the autophagy machinery might provide a scaffold for the formation of a DISC using co-immunoprecipitation. While the DISC proteins, RIPK3 and FADD coimmunoprecipitated with RIPK1 in uninfected macrophages and HIV-M $\phi$, CASP8 and MLKL only coimmunoprecipitated with RIPK1 in HIV-M $\phi$ (Fig. 6b). Also in $\operatorname{HIV}-\mathrm{M} \phi$, proteins involved in early-to-mid stages of autophagy including ATG5, ATG7, and SQSTM1 co-immunoprecipitated with RIPK1 (Fig. 6b), while proteins that are important for earlier signaling in the autophagy process (BECN1 and ULK1 (unc-51-like autophagy-activating kinase 1)) and those involved in later steps, when autophagosomes fuse with lysosomes (syntaxin 17 [STX17] and lysosomal-associated membrane protein 1 (LAMP1)) did not. 


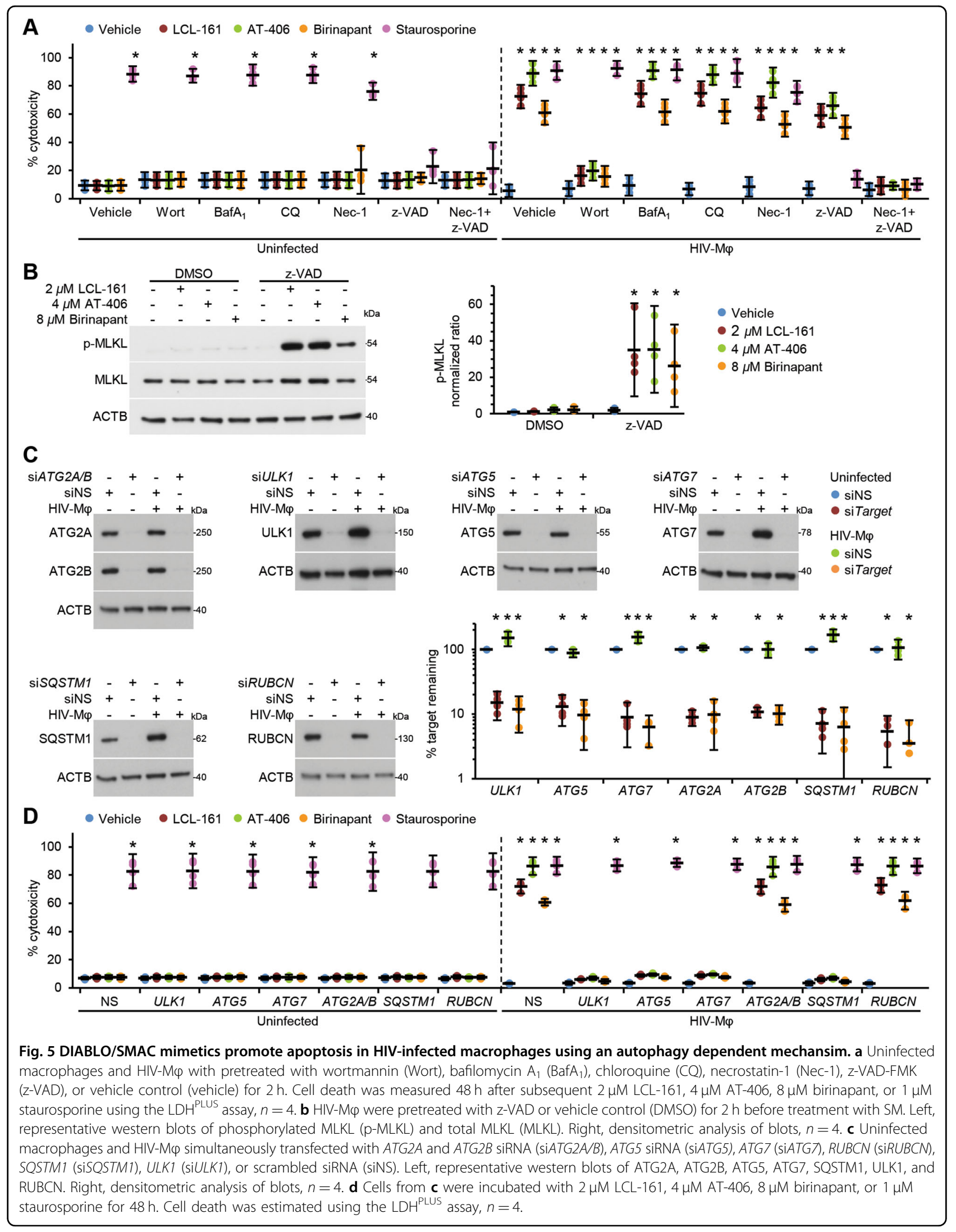




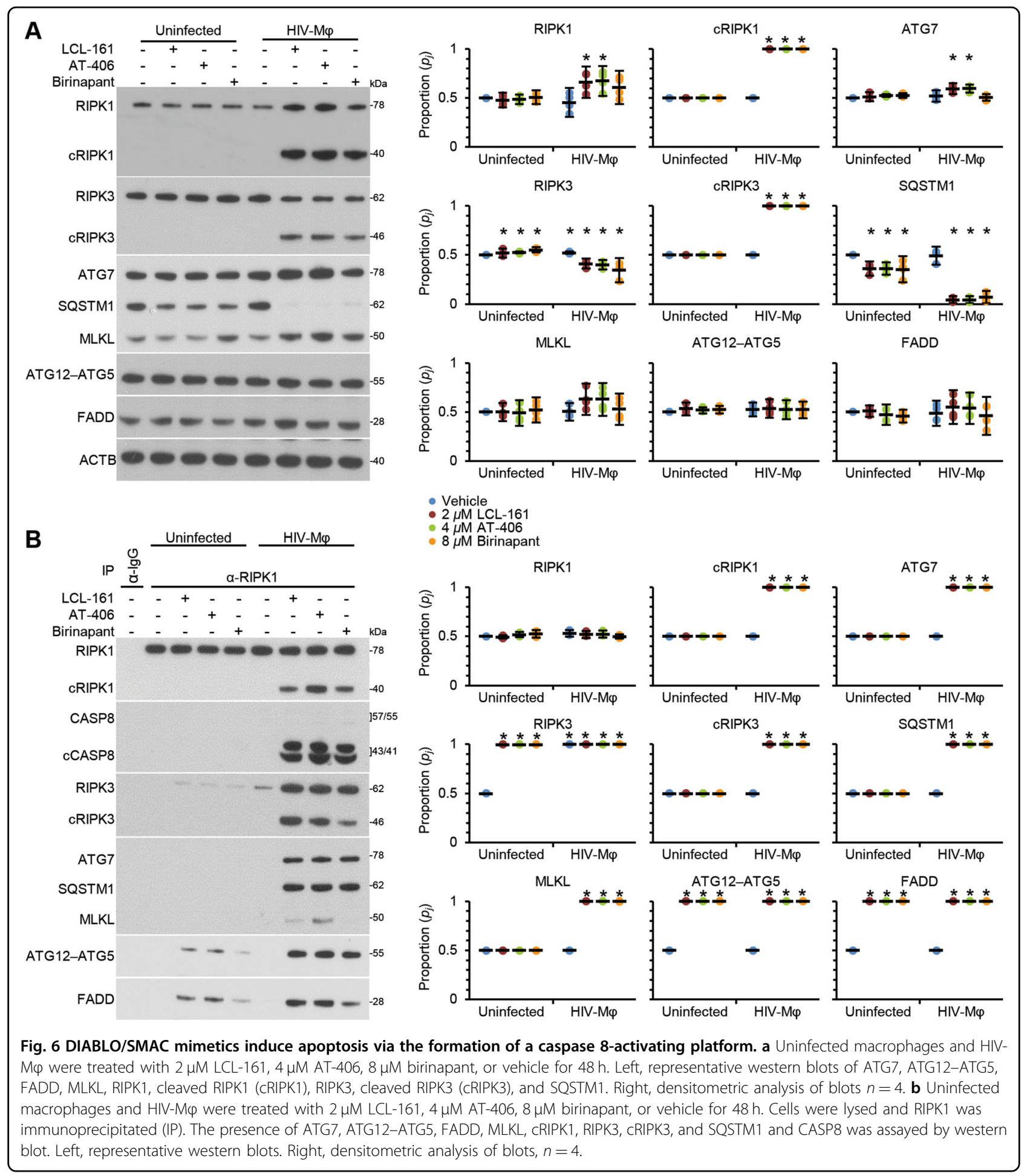

We next assessed whether the autophagy proteins ATG5 and ATG7 are required for efficient SM-mediated DISC formation and activation in HIV-M $\phi$ using RNAi. As RIPK1 directly interacts with SQSTM1 (ref. ${ }^{40}$ ), we also silenced for SQSTM1. Silencing for ATG5, ATG7, or SQSTM1 each ablated the SM-mediated CASP8,
RIPK1, and RIPK3 cleavage (Fig. 7a, Supplementary Fig. 2) and induced a significant negative change in CASP8, RIPK3, MLKL, ATG7, ATG5, FADD, and SQSTM1 coimmunoprecipitation with RIPK1 (Fig. 7b, Supplementary Fig. 3). Notably, MLKL did not associate with RIPK1 after any RNAi. These findings correspond to the increase in 

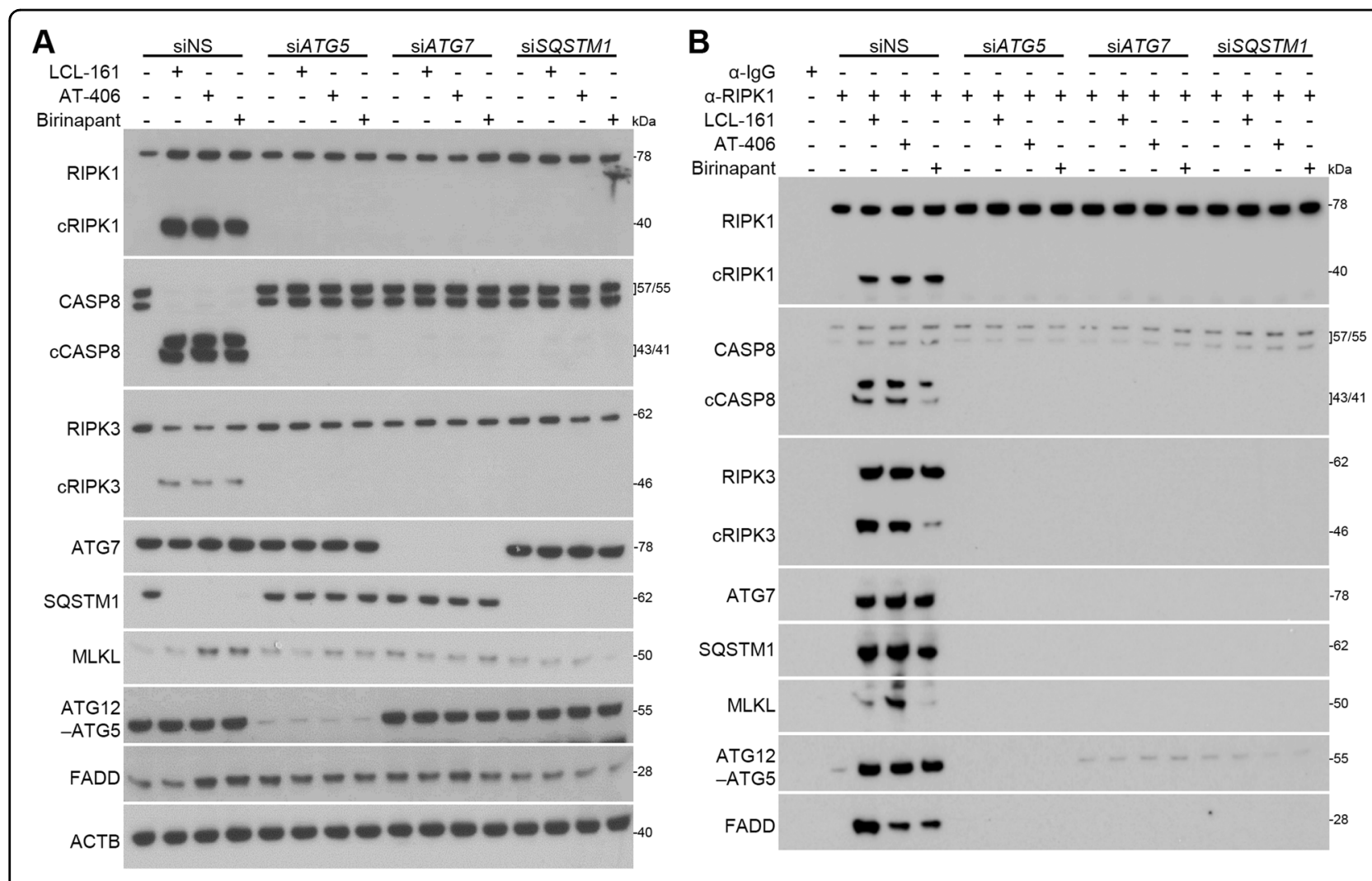

Fig. 7 DIABLO/SMAC mimetics induce apoptosis via an autophagy-dependent caspase 8 activating platform. HIV-M $\varphi$ transfected with $A T G_{5}$

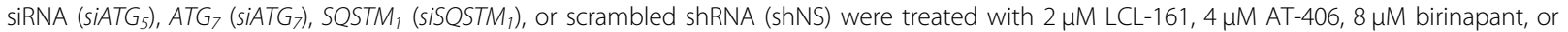
vehicle for $48 \mathrm{~h}$. a Representative western blots of ATG7, ATG12-ATG5, FADD, MLKL, RIPK1, cleaved RIPK1 (cRIPK1), RIPK3, cleaved RIPK3 (cRIPK3), and SQSTM1, $n=4$. Densitometric analysis of blots is shown in Supplementary Fig. 2. b Cells were lysed and RIPK1 was immunoprecipitated (IP). The presence of ATG7, ATG12-ATG5, FADD, MLKL, CRIPK1, RIPK3, CRIPK3, and SQSTM1 and CASP8 in immunoprecipitates was assayed by western blot, $n=4$. Densitometric analysis of blots is shown in Supplementary Fig. 3.

cell viability observed upon SM treatment of ATG5, ATG7, and SQSTM1-silenced cells (Fig. 5d). Collectively, these data indicate that SM require ATG5, ATG7, and SQSTM1 for the assembly and activation of a DISC in HIV-M $\phi$.

As RIPK1 directly interacts with SQSTM1, it is possible that DISC components translocate to autophagosomal membranes. Therefore, to determine the stage of autophagosome formation at which DISCs localize to autophagic structures, we analyzed membranes in $A T G 2 A / B$-silenced cells using differential centrifugation, using LC3B-II and SQSTM1 as markers of autophagosome membranes and autophagy substrates, respectively ${ }^{41}$. In the control cells, and in the absence of SM, LC3B (as LC3B-I), SQSTM1, FADD, and RIPK1 were located primarily in the cytosol (the high-speed supernatant (HSS) fraction; Fig. 8). However, in the presence of SM and bafilomycin $\mathrm{A}_{1}, \mathrm{LC} 3 \mathrm{~B}$ and SQSTM1accumulated in the low-speed pellet (LSP) and was resistant to proteinase $\mathrm{K}$ but sensitive to Triton X-100, indicating that they were enclosed in autophagosomes. Conversely, SQSTM1, RIPK1, cleaved RIPK1, and FADD accumulated in the high-speed pellet (HSP) that was sensitive to both proteinase $\mathrm{K}$ and Triton $\mathrm{X}-100$, indicating that these were not enclosed structures. In $A T G 2 A / B$ silenced cells proteinase K-sensitive LC3B-II and SQSTM1 accumulated in the HSP fraction in the absence of birinapant and bafilomycin $A_{1}$. In the presence of $S M$ and bafilomycin $\mathrm{A}_{1}$, LC3B-II and SQSTM1 accumulated in both the LSP and HSP fractions, while proteinase Ksensitive RIPK1, cleaved RIPK1, and FADD were present only in the HSP fraction (Fig. 8). Collectively, this suggests that ATG2A and ATG2B are required for complete closure of autophagosomes, as LC3B-II-positive structures accumulate in their absence ${ }^{41}$, and that RIPK1 and FADD do not translocate into closed autophagosomes after SM treatment.

\section{Discussion}

Although ART has greatly improved life expectancy and quality for those infected with HIV, multidrug resistance continues to increase, emphasizing the importance of identifying novel strategies to improve HIV treatment 


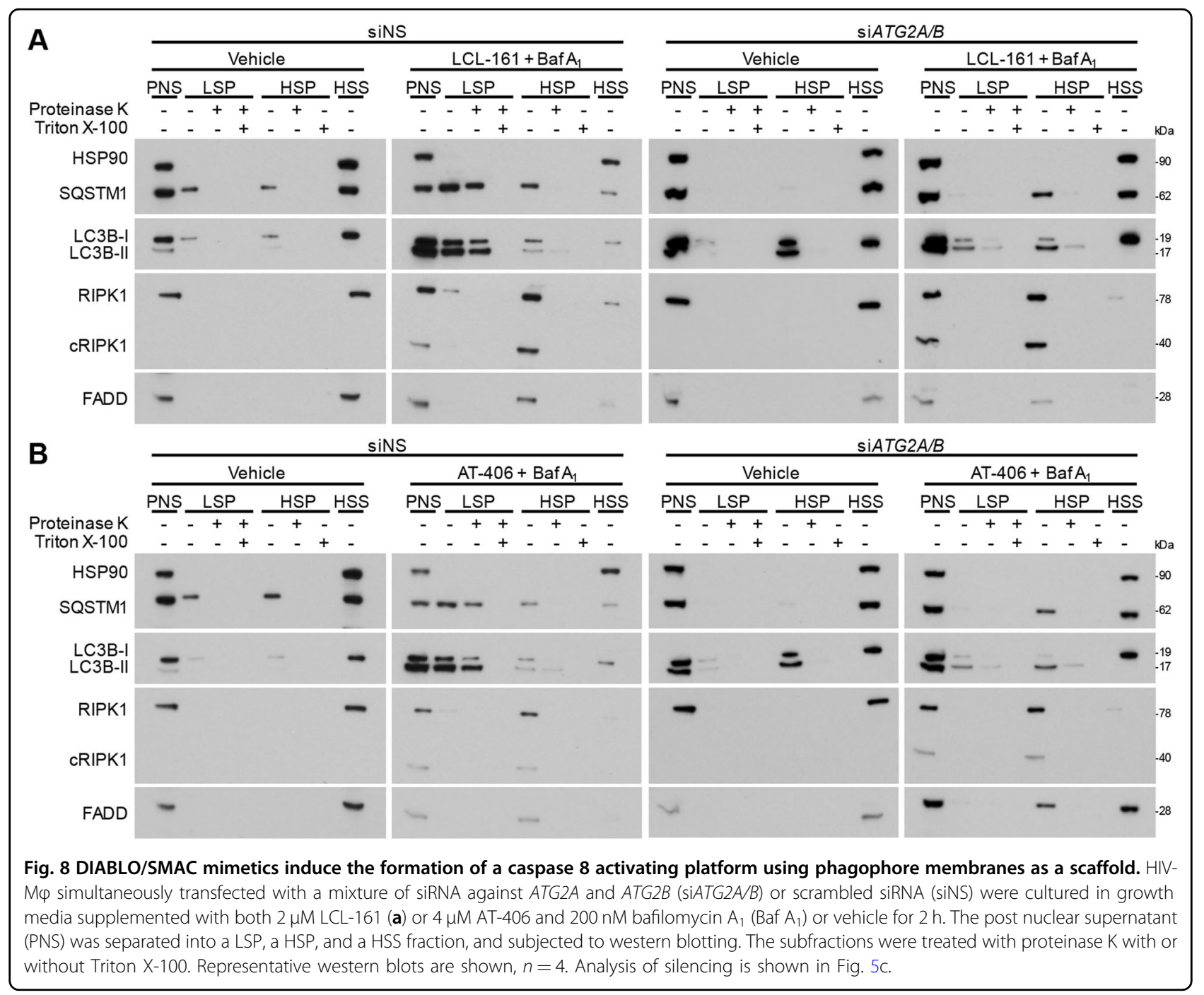

with the goal of reducing the size of the viral reservoir. Although current efforts to purge the latent HIV reservoir predominantly endeavor to reactivate viral production from latently infected $\mathrm{CD} 4+\mathrm{T}$ cells followed by immunologic suppression or viral clearance through ART, this strategy has, to date, failed to decrease significantly the viral reservoir ${ }^{42-46}$. This approach will be even less effective in macrophages since HIV-infected macrophages are particularly resistant to HIV-mediated apoptosis and CD8+ T-cell-mediated killing ${ }^{8-10}$. Despite the documented importance of macrophages, few studies have targeted killing HIV-infected macrophages as part of a cure strategy, although some show promise $\mathrm{e}^{21,47-49}$.

In the present study, we used an alternative strategy to activate cell death pathways selectively in HIV-infected macrophages, while sparing uninfected cells. Similar to our data with HIV-T ${ }_{\mathrm{CM}}{ }^{19}$, the selective SM-mediated apoptosis of HIV-M $\phi$ is dependent upon the induction of autophagy and the translocation, and interaction of FADD and the CASP8 homocomplex with both ATG5 and SQSTM1-self-oligomerized SQSTM1 localizes and recruits RIPK1 to the endoplasmic reticulum-associated autophagosome formation site independently of LC3 and is retained as autophagosomes mature ${ }^{50,51}$, while CASP8 and RIPK1 tethering to autophagosomal membranes involves ATG5 and FADD ${ }^{52}$. We also show that in HIV$\mathrm{M} \phi$, RNAi and pharmacological inhibition of early autophagy events inhibits the SM-mediated formation of a cytosolic DISC, CASP8 activation, and subsequent cell death, whereas neither simultaneous silencing of $A T G 2 A$ and $A T G 2 B$, nor the pharmacological inhibition of autophagosome-lysosome fusion protected cells from SM-mediated cell death. Moreover, RIPK1 and FADD co-localize with SQSTM1 in the HSP. Collectively these data suggest that autophagy proteins serve as a platform for the assembly of a cytosolic DISC that initiates apoptosis, linking autophagy to the control of cell survival and death. 
Importantly, two of the SM tested, LCL-161 and birinapant, failed to increase the viral production from HIV$\mathrm{M} \phi$. These findings correlate with previous studies that showed that the SM SBI-0637142, LCL-161, GDC-0152, and birinapant all failed to activate latent provirus in resting $\mathrm{CD} 4+\mathrm{T}$ cells collected from HIV-infected patients undergoing $\mathrm{ART}^{19,53}$. Conversely, AT-406 increased the production of HIV p24 from HIV-M $\phi$ in agreement with Bobardt et al. ${ }^{54}$. This increase correlated with increased TNF production not observed with either LCL-161 or birinapant treatment.

During HIV infection, macrophages are primary producers of TNF, express constitutively activated NFkB, upregulate IAPs, and are resistant to many apoptotic stimuli, including TNF superfamily members ${ }^{55,56}$. In this state, binding of TNF to its receptor leads to the activation of TNF complex I, comprised of TRADD, the ubiquitin ligases TRAF2 and TRAF5, IKBKG (NEMO), and TNF alpha-induced protein 3 together with the linear ubiquitin chain assembly complex, RIPK1, BIRC2, and BIRC3. In addition to binding caspases, BIRC2 also interacts with TRAF2, which mediates their recruitment to TNFRSF1A. The BIR3 domain of BIRC2 binds RIPK1, while its E3 ligase activity mediates the ubiquitination of RIPK1, stimulating $\mathrm{NFKB}$ activation and suppressing activation of CASP8, ultimately leading to pro-survival signaling ${ }^{25,57,58}$. After SM-induced degradation of IAPs, the TNF-receptor ligation initiates the formation of a DISC known as TNF complex IIa, which leads to the cleavage and activation of CASP8 and the induction of apoptosis, resulting in the reversal of the role of TNF from promoting survival to inducing cell death ${ }^{59}$. The evidence for this occurring in SM-treated HIV-M $\phi$ stems from the TNF neutralization experiment that demonstrated that neutralizing TNF completely ablated the effect of SM, indicating the dependency of SM-mediated apoptosis of HIV-M $\phi$ on TNF (Fig. 2e). This is in contrast to what we found in HIV-T $\mathrm{T}_{\mathrm{CM}}$ where $\mathrm{SM}$-mediated apoptosis was independent of $\mathrm{TNF}^{19}$. However, despite the initiation of apoptosis and the involvement of caspases, we were unable to block cell death using the pan-caspase inhibitor z-VAD-FMK. In this case, when we reduced IAP activity through treatment with SM while inhibiting caspases, MLKL became activated, and the cells died through caspase-independent necroptosis (Fig. $5 \mathrm{~b})^{30}$. Therefore, SM can potentiate TNF-stimulated cell death of HIV-M $\phi$ by promoting either apoptosis or necroptosis in a context-dependent manner. Thus, for the purposes of sensitizing HIV-M $\phi$ to the cytotoxic effects of HIV, IAPs are critical targets.

In summary, our data identify a molecular mechanism whereby we can selectively induce apoptosis in HIVinfected macrophages through a SM-mediated autophagy-dependent assembly of a DISC. Our study thus highlights IAPs as therapeutic targets to clear the macrophage HIV reservoir, and suggests that current HIV cure strategies should include the targeting of IAPs in efforts to achieve HIV eradication.

\section{Materials and methods Macrophages}

Venous blood was drawn from human subjects using protocols approved by the Human Research Protections Program of the University of California San Diego in accordance with the requirements of the Code of Federal Regulations on the Protection of Human Subjects (45 CFR 46 and 21 CFR 50 and 56). All volunteers gave written informed consent prior to their participation. PBMC were isolated from whole blood by density gradient centrifugation over Ficoll-Paque Plus (GE Healthcare). Monocyte derived macrophages were prepared from PBMC by adherence, using colony stimulating factor 1 (Peprotech) as previously described $^{21}$.

\section{HIV}

The following were obtained from the NIH AIDS Reagent Program: HIV $\mathrm{Ba}_{\mathrm{L}-\mathrm{L}}$ from Suzanne Gartner, Mikulas

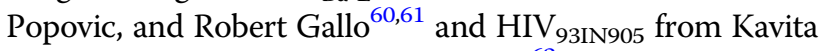
Lole, Robert Bollinger, and Stuart Ray ${ }^{62}$. Virus stocks were prepared as previously described ${ }^{19,63}$. Macrophages were infected with HIV at 0.04 multiplicity of infection for $4 \mathrm{~h}$, washed then cultured for 10 days before use in experiments as previously described ${ }^{21,64}$.

\section{Cell death assays}

Apoptotic ssDNA damage measurements were made using the ssDNA Apoptosis ELISA Kit (Millipore) as described previously ${ }^{65}$. LDH activity in cell supernatants was measured using a mixture of diaphorase $/ \mathrm{NAD}^{+}$and 3-(4-iodophenyl)-2-(4-nitrophenyl)-5-phenyl-2H-tetrazol-3-ium chloride/sodium 2-hydroxypropanoate using the $\mathrm{LDH}^{\text {PLUS }}$ assay (Roche). Percent cytotoxicity was calculated according to the manufacturer's protocol using a LDH standard curve and a cell lysate standard curve.

\section{Chemicals}

LCL-161, AT-406, birinapant (Selleck Chemicals) and staurosporine (Sigma) were prepared in dimethyl sulfoxide. Corresponding volumes of DMSO $(5 \mu \mathrm{L}$ DMSO $\mathrm{mL}^{-1}$ ) were used for vehicle in each experiment. For inhibitor experiments, $20 \mu \mathrm{M}$ z-VAD-FMK, $200 \mathrm{nM}$ wortmannin, $10 \mu \mathrm{M}$ chloroquine, $10 \mathrm{nM}$ bafilomycin $\mathrm{A}_{1}$, or $10 \mu \mathrm{M}$ necrostatin-1 (all Sigma) were added for $1 \mathrm{~h}$ prior to, and for the duration of the experiment. The human TNF neutralizing antibody (D1B4; Cell Signaling) was added $2 \mathrm{~h}$ prior to $\mathrm{SM}$ treatment at $1 \mu \mathrm{g} \mathrm{mL} \mathrm{m}^{-1}$ as previously described ${ }^{19}$. 


\section{siRNA transfection}

Macrophages were transfected with ThermoFisher Silencer Select $A T G 2 A$ (ID\# s23098), ATG2B (ID\# s30173), ATG5 (ID\# s18159), ATG7 (ID\# s20651), RUBCN (ID\# s18718), SQSTM1 (ID\# s16961), ULK1 (ID\# s15965), or control (Cat\# 4390846) siRNA (siNS), using lipofectamine RNAiMAX transfection reagent (ThermoFisher) in Opti-MEM (Gibco) according to the manufacturer's instructions. Forty eight hours later, cells were analyzed for target gene silencing and used in experiments. Transfection efficiency was assessed with BLOCK-iT Alexa Fluor Red Fluorescent Control (ThermoFisher) using flow cytometry ${ }^{19}$.

\section{Western blotting}

ATG2A (\#15011), ATG5 (\#2630), ATG7 (\#2631), BECN1 (\#3738), BIRC2 (D5G9), CASP8 (1C12), FADD (\#2782), FAS (C18C12), FASLG (\#4273), HSP90 (C45G5); LAMP1 (C54H11), MLKL (D2I6N), PARP1 (46D11), RIPK1 (D94C12), RIPK3 (D4G2A), ULK1 (D9D7), and XIAP (3B6) antibodies were obtained from Cell Signaling. SQSTM1 (\#ab56416) and STX17 (\#ab116113) antibodies were purchased from Abcam. $\beta$-actin (ACTB; AC-74) and ATG2B (\#HPA019665) were purchased from Sigma. MAP1LC3B (\#NB100-2220) was purchased from Novus Biologicals. Cell lysis, co-immunoprecipitation, subcellular fractionation, and western blotting were performed as previously described $^{19,41}$. Relative densities of the target bands to the reference bands was calculated using Image (NIH).

\section{Statistics}

Samples were assigned to experimental groups through simple random sampling. Sample size $(n)$ was determined using a two-sample two-sided equality test with power $(1-\beta)=0.8, \alpha=0.05$, and preliminary data where the minimum difference in outcome was at least $70 \%$. Data are represented as dot blots with arithmetic means $\pm 95 \%$ confidence interval for independent biological replicates. Data were assessed for symmetry, or skewness, using Pearson's skewness coefficient. Normalized ratiometric data were $\log _{2}$ transformed. When expression in the reference sample is low or zero, we used proportion statistics as described previously ${ }^{19,66}$. Comparisons between groups were performed using the paired, two-tailed, Student's $t$-test. In all experiments, differences were considered significant when $P<0.05$; ${ }^{*} P<0.05$.

\section{Acknowledgements}

This work was supported, in whole or in part, by the National Institute of Neurological Disorders and Stroke of the NIH (www.ninds.nih.gov; R01 NS104015 to S.A.S.), and the International Maternal Pediatric Adolescent AIDS Clinical Trials Network (impaactnetwork.org). Overall support for the International Maternal Pediatric Adolescent AIDS Clinical Trials (IMPAACT) Network is provided by the National Institute of Allergy and Infectious Diseases (NIAID) of the NIH under award numbers UM1AI068632 (IMPAACT LOC), UM1AI068616 (IMPAACT SDMC), and UM1AI106716 (IMPAACT LC), with co-funding from the Eunice Kennedy Shriver National Institute of Child Health and Human Development (NICHD) and the National Institute of Mental Health (NIMH). The content is solely the responsibility of the authors and does not necessarily represent the official views of the $\mathrm{NIH}$. The funders had no role in study design, data collection and analysis, decision to publish, or preparation of the manuscript.

\section{Author details}

${ }^{1}$ Division of Infectious Diseases, Department of Pediatrics, University of California San Diego, La Jolla, CA, USA. ${ }^{2}$ Rady Children's Hospital, San Diego, CA, USA. ${ }^{3}$ Present address: Chicago Medical School, Rosalind Franklin University of Medicine and Science, North Chicago, IL, USA. ${ }^{4}$ Present address: Gladstone Center for HIV Cure Research, Gladstone Institute of Virology and Immunology, San Francisco, CA, USA

\section{Conflict of interest}

The authors declare that they have no conflict of interest.

\section{Publisher's note}

Springer Nature remains neutral with regard to jurisdictional claims in published maps and institutional affiliations.

Supplementary Information accompanies this paper at (https://doi.org/ 10.1038/s41419-020-02761-x).

Received: 27 March 2020 Revised: 29 June 2020 Accepted: 6 July 2020 Published online: 27 July 2020

\section{References}

1. Igarashi, T. et al. Macrophage are the principal reservoir and sustain high virus loads in rhesus macaques after the depletion of CD4 $+T$ cells by a highly pathogenic simian immunodeficiency virus/HIV type 1 chimera (SHIV): Implications for HIV-1 infections of humans. Proc. Natl Acad. Sci. USA 98 658-663 (2001).

2. Abbas, W., Tariq, M., Iqbal, M., Kumar, A. \& Herbein, G. Eradication of HIV1 from the macrophage reservoir: an uncertain goal? Viruses 7, 1578-1598 (2015)

3. Ganor, Y. et al. HIV-1 reservoirs in urethral macrophages of patients under suppressive antiretroviral therapy. Nat. Microbiol. 4, 633-644 (2019).

4. Honeycutt, J. B. et al. Macrophages sustain HIV replication in vivo independently of T cells. J. Clin. Invest. 126, 1353-1366 (2016).

5. Honeycutt, J. B. et al. HIV persistence in tissue macrophages of humanized myeloid-only mice during antiretroviral therapy. Nat. Med. 23 638-643 (2017).

6. Cory, T. J., Schacker, T. W., Stevenson, M. \& Fletcher, C. V. Overcoming pharmacologic sanctuaries. Curr. Opin. HIV Aids 8, 190-195 (2013).

7. Duncan, C. J. et al. High-multiplicity HIV-1 infection and neutralizing antibody evasion mediated by the macrophage-T cell virological synapse. J. Virol. 88, 2025-2034 (2014)

8. Abbas, W. \& Herbein, G. Plasma membrane signaling in HIV-1 infection. Biochim. Biophys. Acta 1838, 1132-1142 (2014).

9. Clayton, K. L. et al. Resistance of HIV-infected macrophages to CD8(+) T lymphocyte-mediated killing drives activation of the immune system. Nat. Immunol. 19, 475-486 (2018).

10. Boliar, S. et al. Inhibition of the IncRNA SAF drives activation of apoptotic effector caspases in HIV-1-infected human macrophages. Proc. Natl Acad. Sci. USA 116, 7431-7438 (2019).

11. Wolf, D. et al. HIV-1 Nef associated PAK and PI3-kinases stimulate Aktindependent Bad-phosphorylation to induce anti-apoptotic signals. Nat. Med. 7, 1217-1224 (2001)

12. Guillemard, E. et al. Human immunodeficiency virus 1 favors the persistence of infection by activating macrophages through TNF. Virology 329, 371-380 (2004).

13. Berro, R. et al. Identifying the membrane proteome of HIV-1 latently infected cells. J. Biol. Chem. 282, 8207-8218 (2007).

14. Swingler, S., Mann, A. M., Zhou, J., Swingler, C. \& Stevenson, M. Apoptotic killing of HIV-1-infected macrophages is subverted by the viral envelope glycoprotein. PLoS Pathog. 3, 1281-1290 (2007). 
15. Campbell, G. R. \& Loret, E. P. What does the structure-function relationship of the HIV-1 Tat protein teach us about developing an AIDS vaccine? Retrovirology 6, 50 (2009)

16. López-Huertas, M. R. et al. The presence of HIV-1 Tat protein second exon delays fas protein-mediated apoptosis in CD4+ T lymphocytes: a potential mechanism for persistent viral production. J. Biol. Chem. 288, 7626-7644 (2013).

17. Denner, J. et al. Modulation of cytokine release and gene expression by the immunosuppressive domain of gp41 of HIV-1. PLOS ONE 8, e55199 (2013).

18. Yuan, Z. et al. HIV-related proteins prolong macrophage survival through induction of triggering receptor expressed on myeloid cells-1. Sci. Rep. 7, 42028 (2017).

19. Campbell, G. R., Bruckman, R. S., Chu, Y. L., Trout, R. N. \& Spector, S. A. SMAC mimetics induce autophagy-dependent apoptosis of HIV-1-infected resting memory CD4+ T cells. Cell Host Microbe 24, 689-702.e687 (2018).

20. Hyun, J. et al. HIV and HCV augments inflammatory responses through increased TREM-1 expression and signaling in Kupffer and Myeloid cells. PLOS Pathog. 15, e1007883 (2019).

21. Campbell, G. R., To, R. K. \& Spector, S. A. TREM-1 protects HIV-1-infected macrophages from apoptosis through maintenance of mitochondrial function. mBio 10, e02638-02619 (2019).

22. Wang, X., Ragupathy, V., Zhao, J. \& Hewlett, I. Molecules from apoptotic pathways modulate HIV-1 replication in Jurkat cells. Biochem. Biophys. Res. Commun. 414, 20-24 (2011).

23. Gao, Z. et al. A dimeric Smac/diablo peptide directly relieves caspase-3 inhibition by XIAP. Dynamic and cooperative regulation of XIAP by Smac/Diablo. J. Biol. Chem. 282, 30718-30727 (2007).

24. Varfolomeev, E. et al. IAP antagonists induce autoubiquitination of c-IAPs, NFkappaB activation, and TNFalpha-dependent apoptosis. Cell 131, 669-681 (2007).

25. Bertrand, M. J. et al. CIAP1 and CIAP2 facilitate cancer cell survival by functioning as E3 ligases that promote RIP1 ubiquitination. Mol. Cell. 30, 689-700 (2008).

26. Weisberg, E. et al. Smac mimetics: implications for enhancement of targeted therapies in leukemia. Leukemia 24, 2100-2109 (2010).

27. Cai, Q. et al. A potent and orally active antagonist (SM-406/AT-406) of multiple inhibitor of apoptosis proteins (IAPS) in clinical development for cancer treatment. J. Med. Chem. 54, 2714-2726 (2011).

28. Allensworth, J. L., Sauer, S. J., Lyerly, H. K., Morse, M. A. \& Devi, G. R. Smac mimetic Birinapant induces apoptosis and enhances TRAIL potency in inflammatory breast cancer cells in an IAP-dependent and TNF-alpha-independent mechanism. Breast Cancer Res. Treat. 137, 359-371 (2013).

29. Vince, J. E. et al. IAP antagonists target CIAP1 to induce TNFalpha-dependent apoptosis. Cell 131, 682-693 (2007)

30. Wang, L., Du, F. \& Wang, X. TNF-alpha induces two distinct caspase-8 activation pathways. Cell 133, 693-703 (2008)

31. Lu, J. et al. SM-164: a novel, bivalent Smac mimetic that induces apoptosis and tumor regression by concurrent removal of the blockade of CIAP-1/2 and XIAP. Cancer Res. 68, 9384-9393 (2008).

32. Frankfurt, O. S. \& Krishan, A. Enzyme-linked immunosorbent assay (ELISA) for the specific detection of apoptotic cells and its application to rapid drug screening. J. Immunol. Methods 253, 133-144 (2001).

33. Fulda, S. Promises and challenges of SMAC mimetics as cancer therapeutics. Clin. Cancer Res. 21, 5030-5036 (2015).

34. Petersen, S. L. et al. Autocrine TNFalpha signaling renders human cancer cells susceptible to Smac-mimetic-induced apoptosis. Cancer Cell. 12, 445-456 (2007).

35. Klionsky, D. J. et al. Guidelines for the use and interpretation of assays for monitoring autophagy (3rd edition). Autophagy 12, 1-222 (2016).

36. Yoon, S., Bogdanov, K., Kovalenko, A. \& Wallach, D. Necroptosis is preceded by nuclear translocation of the signaling proteins that induce it. Cell Death Differ. 23, 253-260 (2016).

37. Moquin, D. \& Chan, F. K. The molecular regulation of programmed necrotic cell injury. Trends Biochem. Sci. 35, 434-441 (2010).

38. Feoktistova, M. et al. CIAPs block ripoptosome formation, a RIP1/caspase-8 containing intracellular cell death complex differentially regulated by cFLIP isoforms. Mol. Cell 43, 449-463 (2011).

39. Tenev, T. et al. The ripoptosome, a signaling platform that assembles in response to genotoxic stress and loss of IAPs. Mol. Cell 43, 432-448 (2011).
40. Sanz, L., Sanchez, P., Lallena, M. J., Diaz-Meco, M. T. \& Moscat, J. The interaction of p62 with RIP links the atypical PKCs to NF-kappaB activation. EMBO J. 18 3044-3053 (1999)

41. Velikkakath, A. K., Nishimura, T., Oita, E., Ishihara, N. \& Mizushima, N. Mammalian Atg2 proteins are essential for autophagosome formation and important for regulation of size and distribution of lipid droplets. Mol. Biol. Cell 23, 896-909 (2012).

42. Shan, L. et al. Stimulation of HIV-1-specific cytolytic T lymphocytes facilitates elimination of latent viral reservoir after virus reactivation. Immunity $\mathbf{3 6}$ 491-501 (2012).

43. Bullen, C. K., Laird, G. M., Durand, C. M., Siliciano, J. D. \& Siliciano, R. F. New ex vivo approaches distinguish effective and ineffective single agents for reversing HIV-1 latency in vivo. Nat. Med. 20, 425-429 (2014).

44. Laird, G. M. et al. Ex vivo analysis identifies effective HIV-1 latency-reversing drug combinations. J. Clin. Invest. 125, 1901-1912 (2015).

45. Spivak, A. M. \& Planelles, V. HIV-1 eradication: early trials (and tribulations). Trends Mol. Med. 22, 10-27 (2016).

46. Walker-Sperling, V. E., Pohlmeyer, C. W., Tarwater, P. M. \& Blankson, J. N. The effect of latency reversal agents on primary CD8+ T cells: Implications for shock and kill strategies for human immunodeficiency virus eradication. EBioMedicine 8, 217-229 (2016).

47. Cunyat, F. et al. Colony-stimulating factor 1 receptor antagonists sensitize human immunodeficiency virus type 1-infected macrophages to TRAILmediated killing. J. Virol. 90, 6255-6262 (2016).

48. Xue, J. et al. Galectin-3 promotes caspase-independent cell death of HIV-1infected macrophages. FEBS J. 284, 97-113 (2017).

49. Zhang, G., Luk, B. T., Hamidy, M., Zhang, L. \& Spector, S. A. Induction of a Na $(+) / K(+)$-ATPase-dependent form of autophagy triggers preferential cell death of human immunodeficiency virus type-1-infected macrophages. Autophagy 14, 1359-1375 (2018).

50. Itakura, E. \& Mizushima, N. p62 Targeting to the autophagosome formation site requires self-oligomerization but not LC3 binding. J. Cell Biol. 192, 17-27 (2011).

51. Goodall, M. L. et al. The autophagy machinery controls cell death switching between apoptosis and necroptosis. Dev. Cell. 37, 337-349 (2016).

52. Bell, B. D. et al. FADD and caspase-8 control the outcome of autophagic signaling in proliferating T cells. Proc. Natl Acad. Sci. USA 105, 16677-16682 (2008).

53. Pache, L. et al. BIRC2/CIAP1 is a negative regulator of HIV-1 transcription and can be targeted by Smac mimetics to promote reversal of viral latency. Cell Host Microbe 18, 345-353 (2015).

54. Bobardt, M. et al. The inhibitor apoptosis protein antagonist Debio 1143 Is an attractive HIV-1 latency reversal candidate. PLOS ONE 14, e0211746 (2019).

55. Lee, C., Tomkowicz, B., Freedman, B. D. \& Collman, R. G. HIV-1 gp120-induced TNF-a production by primary human macrophages is mediated by phosphatidylinositol-3 (PI-3) kinase and mitogen-activated protein (MAP) kinase pathways. J. Leukoc. Biol. 78, 1016-1023 (2005).

56. Leghmari, K., Contreras, X., Moureau, C. \& Bahraoui, E. HIV-1 Tat protein induces TNF-alpha and IL-10 production by human macrophages: differential implication of PKC-betall and -delta isozymes and MAP kinases ERK1/2 and p38. Cell Immunol. 254, 46-55 (2008)

57. Vanlangenakker, N., Vanden Berghe, T. \& Vandenabeele, P. Many stimuli pull the necrotic trigger, an overview. Cell Death Differ. 19, 75-86 (2012).

58. Afonina, I. S., Zhong, Z., Karin, M. \& Beyaert, R. Limiting inflammation-the negative regulation of NF-kappaB and the NLRP3 inflammasome. Nat. Immunol. 18, 861-869 (2017).

59. Holbrook, J., Lara-Reyna, S., Jarosz-Griffiths, H. \& McDermott, M. Tumour necrosis factor signalling in health and disease. F1000Res. 8, 111 (2019).

60. Gartner, S. et al. The role of mononuclear phagocytes in HTLV-III/LAV infection. Science 233, 215-219 (1986).

61. Popovic, M., Gartner, S., Read-Connole, E., Beaver, B., Reitz, M. in Retroviruses of Human AIDS and Related Animal Diseases, Colloque Des Cent Gardes, Vol. 3 (eds Girard, M. \& Valette, L.) 21-27 (Pasteur Vaccins: Marnes-La-Coquette, France, 1988).

62. Lole, K. S. et al. Full-length human immunodeficiency virus type 1 genomes from subtype C-infected seroconverters in India, with evidence of intersubtype recombination. J. Virol. 73, 152-160 (1999).

63. Campbell, G. R., Loret, E. P. \& Spector, S. A. HIV-1 clade B Tat, but not clade C Tat, increases X4 HIV-1 entry into resting but not activated CD4+ T cells. J. Biol. Chem. 285, 1681-1691 (2010). 
64. Campbell, G. R. \& Spector, S. A. Vitamin D inhibits human immunodeficiency virus type 1 and Mycobacterium tuberculosis infection in macrophages through the induction of autophagy. PLoS Pathog. 8, e1002689 (2012).

65. Campbell, G. R., Watkins, J. D., Loret, E. P. \& Spector, S. A. Differential induction of rat neuronal excitotoxic cell death by human immunodeficiency virus type 1 clade B and C Tat proteins. AIDS Res Hum. Retroviruses 27, 647-654 (2011).

66. Bergemann, T. L. \& Wilson, J. Proportion statistics to detect differentially expressed genes: a comparison with log-ratio statistics. BMC Bioinformatics 12, 228 (2011). 